\title{
Auction-Based Scheduling in Non-Cooperative Multiuser OFDM Systems
}

\author{
Zhen Kong \\ Department of EEE \\ The University of Hong Kong \\ Hong Kong, China \\ zkong@eee.hku.hk
}

\author{
Yu-Kwong Kwok \\ Department of ECE \\ Colorado State University \\ Fort Collins, CO 80523, USA \\ Ricky.Kwok@colostate.edu
}

\author{
Jiangzhou Wang \\ Department of Electronics \\ University of Kent \\ Canterbury, Kent, UK \\ j.z.wang@kent.ac.uk
}

\begin{abstract}
We study the problem of achieving proportional fair resource allocation in a non-cooperative multiuser OFDM network. We propose an auction-based scheduling algorithm, which combines the merits of the VCG auction and the greedy MC PF algorithm, to ensure that wireless users truthfully declare their resource requirements even though the users are inherently selfish. Through simulations, we find that users lying about their resource requirements are severely penalized by very high payments so that they should rather declare true valuations of subcarriers to the scheduler. Thus, the proposed auction-based scheduling algorithm can be used efficiently in a non-cooperative situation to realize proportional fairness.
\end{abstract}

Keywords: Auction; VCG mechanism; resource scheduling; OFDM; non-cooperative wireless networks.

\section{INTRODUCTION}

Traditionally, wireless resource management is based on the premise that autonomous wireless users are cooperative so that the precious resource (e.g., bandwidth) can be allocated among users in a globally optimal manner. However, in many practical scenarios, wireless users are autonomous and thus, may exhibit non-cooperative behaviors due to self-interests [1]. For instance, in a public WLAN hot spot, individual users may attempt to deviate from the standard protocols or algorithms and behave in a rational but selfish manner so as to gain advantages in radio resource allocation, without regard to the overall system performance.

Recently, the problem of non-cooperative resource management has become a hot-topic in the research community. For instance, in [1], we have studied the impact of selfish behaviors on efficiency performance for Maximum Rate scheduling algorithm in a non-cooperative TDMA system, and proposed a repeated-game theoretic approach to thwart selfish behaviors and enforce users to report channel conditions truthfully to the scheduler. In many practical situations, besides efficiency, user fairness is also an important factor to consider. It is well known that proportional fairness (PF) [2] has been introduced as an effective solution to achieve a good balance between system throughput and user fairness. On the other hand, some more advanced multi-carrier techniques, such as orthogonal frequency-division multiplexing (OFDM) [3], have also been utilized in current high-speed wireless networks. In this paper we intend to extend our previous work from single-carrier systems to multicarrier systems while achieving PF resource allocation.

There are several difficult challenges to be tackled. Firstly, in this non-cooperative situation, wireless users may be selfish and report their own channel conditions dishonestly as so to maximize their own benefits. As we have shown in [1], such a selfish behavior can dramatically reduce the system throughput. Thus, one of the most important objectives is to design an efficient mechanism to enforce reporting true channel conditions. Secondly, in OFDM systems, multiple subcarriers need to be allocated to different users. This greatly increases the complexity of the scheduling process. Thirdly, the quality of every subcarrier may be different for different users. Indeed, wireless users in OFDM systems compete for multiple units of radio resource instead of single object in the single channel TDMA system. Thus, the resource allocation algorithm must be capable of resolving competition for multiple objects among selfish users. Finally, to be of practical use, the proposed method must be suitable for practical implementation with a low complexity.

Auctions [4] have recently been introduced into several areas of wireless communications to handle the problem of resource competition among selfish users. Sun et al. [5] proposed a channel allocation algorithm based on the second-price auction mechanism to allow wireless users to fairly compete for a wireless fading channel, but it did not consider multicarrier situation. Huang et al. [6] studied auction methods for cooperative communication systems. But whether truthful-revealing is a dominant strategy was not discussed. Fu and Schaar [7] proposed a resource management algorithm based on Vickrey-Clarke-Groves (VCG) mechanism [4] to ensure that wireless users truthfully declare their resource requirements. Gandhi et al. [8] formulated spectrum auctions as multi-unit auctions, and designed a scalable framework to maximize auction revenue and spectrum utilization. However, in their models, they just assumed the qualities of different spectrum bands are identical. Obviously, this is not a practical assumption.

In summary, all these works are about single unit auctions or multi-unit auctions with identical value. But in OFDM systems, the quality of every subcarrier may be different for different users. Thus, the resource allocation problem needs to be formulated as a multi-unit auction with non-identical value, which is usually referred to as combinatorial auction [11]. Pal et al. [9] have proposed a combinatorial reverse auction-based scheduling algorithm to maximize the number of satisfied users in wireless systems. But, the bidding language and computational process are highly complex. The closest work to ours is [10], where an auction algorithm is applied to the OFDMA subcarrier allocation problem. Though it can achieve efficient resource allocation, it cannot prevent 
selfish users from reporting their valuations for subcarriers dishonestly.

In this paper, we consider the design of an auction-based resource allocation algorithm with a low complexity to realize PF in a non-cooperative multiuser OFDM system. In such a system, wireless users compete for subcarriers allocated to them. All users evaluate the valuations of different subcarriers according to their channel conditions and QoS requirements, and submit bids to the scheduler, which also acts as the auctioneer. The auctioneer uses a VCG-based auction method to calculate payment paid by different users so as to enforce selfish users to truthfully report their valuation of subcarriers, and utilizes the greedy MC PF scheduling method proposed in [12] to achieve low-complexity resource allocation with PF in a multiuser OFDM system.

The paper is organized as follows. In Section II, we describe the system model in detail. In Section III, an auction method is explained and presented in detail. Then we present our simulation results in Section IV. Finally, we draw our conclusions in Section V.

\section{MODEL}

Here we consider an OFDM system with $K$ users and $M$ orthogonal frequency subcarriers. The total spectrum bandwidth is $W$. At the physical layer, we use an $L$-path Rayleigh fading channel model. By using adaptive modulation, at time slot $t$, the $k$-th user's data rate (bps) on the $m$-th subcarrier $r_{k, m}^{a}(t)$ can be usually decided by the current channel SNR and the required BER, i.e.,

$$
r_{k, m}^{a}(t)=\left\lfloor\log _{2}\left(1+\frac{-1.5}{\ln \left(5 \cdot P_{b e r}\right)} \cdot \gamma_{k, m}(t)\right)\right\rfloor \cdot \Delta f
$$

where $\gamma_{k, m}(t)$ is the SNR for the $k$-th user's $m$-th subcarrier signal at time instant $t, P_{b e r}$ is the required BER for this transmission, $\Delta f$ be the frequency spacing between two adjacent subcarriers. Let $x_{k, m}(t)$ be the channel assignment status taking value 1 and 0 when the $m$-th subcarrier is and is not occupied by the $k$-th user, then the $k$-th user' instantaneous data rate is defined as:

$$
r_{k}^{a}(t)=\sum_{m=1}^{M} r_{k, m}^{a}(t) \cdot x_{k, m}(t)
$$

In a cooperative situation, the objective of the scheduler is to achieve proportional fairness in multiuser OFDM system. In [12], it has been formulated as a global optimization problem based on the PF definition for MC systems in [13]. Here we redefine it as follows:

$$
\max _{\left\{x_{k, m}\right\}} \sum_{k=1}^{K} \log \left(1+\frac{\sum_{m=1}^{M} x_{k, m}(t) \cdot r_{k, m}^{a}(t)}{\left(t_{c}-1\right) \overline{R_{k}(t)}}\right)
$$

subject to

$$
\text { (I) } \sum_{k=1}^{K} x_{k, m}(t)=1, x_{k, m}(t)=\{0,1\}
$$

where the average rate $\overline{R_{k}(t)}$ can be approximated by a moving average value with average window size $t_{c}$ time slots, i.e.,

$$
\overline{R_{k}(t+1)}=\left(1-\frac{1}{t_{c}}\right) \cdot \overline{R_{k}(t)}+\frac{1}{t_{c}} \cdot r_{k}^{a}(t)
$$

But in a non-cooperative environment, a selfish user may report a bogus channel rate $r_{k, m}(t)$ other than $r_{k, m}^{a}(t)$ or lie about its valuation $v_{k, m}^{a}(t)$ of the radio channel. Without reliable inputs, the resource allocation result is then totally different from that in a cooperative environment. Consequently, the radio resource may be utilized inefficiently.

\section{AUCTION-BASED ALGORITHM}

\section{A. Auction analysis}

Motivated by the above considerations, we set out to solve the problem of resource competition among selfish users by applying the concept of auction from economics. Specifically, an auction is a decentralized market mechanism for allocating resources [7], consisting of three key elements: (1) the good to be allocated; (2) an auctioneer, who determines the allocation of the good and the payment paid by bidders; and (3) a group of bidders, who want to obtain the good from the auctioneer. In the non-cooperative multiuser OFDM system considered in our study, all users compete among each other for the subcarriers and each user has a different valuation for every subcarrier. Now, to formulate this problem as an auction, the good to be allocated are the $M$ subcarriers, the auctioneer is just the scheduler in the BS, and the bidders are the $K$ users.

In a non-cooperative environment, the bidder can untruthfully declare (exaggerate) its resource requirement $r_{k, m}(t)$ or its valuation of subcarriers $v_{k, m}^{a}(t)$ in order to obtain a higher throughput. With inaccurate bidding information, the auctioneer cannot allocate resource among users efficiently and fairly. Thus, we need a mechanism to prevent the bidder from lying to the auctioneer about its valuation for the subcarriers. Indeed, the auction method should be incentive compatible, i.e., a rational bidder will always tell the truth as enticed by the auction rule.

VCG auction [4] has been regarded as one of the most effective mechanism to induce truth-revealing strategies. Specifically, in a VCG auction, by charging each bidder a payment corresponding to the inconvenience it causes to other bidders, the auctioneer can encourage the bidder to report truthfully and thus, resource is allocated in an efficient manner. Thus, we design an auction-based algorithm using the VCG auction model so as to enforce bidders to tell their truthful valuations of the subcarriers.

As described in Section $\mathrm{I}$, another important requirement is to allocate subcarriers among competitive users so as to achieve PF through a multi-unit auction. Most of the existing solutions for multi-unit auctions apply combinatorial auctions as the most general framework [9], [11]. Unfortunately, these auctions usually require complex bid expression that grows exponentially with the size of goods, and depend highly on complex allocation and pricing process, which requires solving NP-hard optimization problems. However, in a practical scheduling scenario, we should avoid the complex combinatorial auctions while achieving PF. 
In our previous study [12], we have proposed a low complexity greedy MC PF method in multiuser OFDM systems. With this method, proportional fairness can be achieved by assigning subcarriers to the user with the highest system PF value one by one. Consequently, the greedy MC PF algorithm can be utilized to allocate subcarriers in real time.

Specifically, in [12], the system PF value if user $k$ occupies subcarrier $m$ is defined as:

$$
\begin{aligned}
& P F(t, k)=\log \left(1+\frac{r_{k}^{\prime}(t)+r_{k, m}^{a}(t)}{\left(t_{c}-1\right) R_{k}(t)}\right)+\sum_{j \neq k} \log \left(1+\frac{r_{j}^{\prime}(t)}{\left(t_{c}-1\right) \cdot \overline{R_{j}(t)}}\right) \\
& =P F(t, k, m)+\sum_{j \neq k} \log \left(1+\frac{r_{j}^{\prime}(t)}{\left(t_{c}-1\right) \cdot \overline{R_{j}(t)}}\right)
\end{aligned}
$$

where $r_{k}^{\prime}(t)$ is the user rate for user $k$ before the assignment of the subcarrier $m$. Because the subcarrier is assigned to the user so that the highest system PF value can be achieved, a rationally selfish user $k$ may just evaluate its valuation $v_{k, m}^{a}(t)$ of subcarriers based on the PF value and report a higher one $v_{k, m}(t)$ so as to increase its opportunity to be scheduled. Then the resulting throughput could be heuristically increased.

Specifically, at time slot $t$, when letting user $k$ 's valuation for sending traffic on subcarrier $m$ with data rate $r_{k, m}^{a}(t)$ be $v_{k, m}^{a}(t)$, it can be expressed by user $k$ 's PF value on subcarrier $m$, i.e.,

$$
v_{k, m}^{a}(t)=P F(t, k, m)=\log \left(1+\frac{r_{k}^{\prime}(t)+r_{k, m}^{a}(t)}{\left(t_{c}-1\right) \overline{R_{k}(t)}}\right)
$$

\section{B. Auction design}

With the above analysis, we can propose an auction-based scheduling algorithm combining the VCG auction, which is employed to calculate payment and realize incentive compatibility, as well as the greedy $\mathrm{MC}$ $\mathrm{PF}$ algorithm, which is used to achieve low-complexity PF scheduling for a multi-user OFDM system. Our algorithm can be summarized as follows.

- Channel condition measurement and valuation:

At the beginning of time slot $t$, every user $k$ measures $r_{k, m}^{a}(t)$ on each subcarrier $m$ corresponding to its experienced SNR $\gamma_{k, m}(t)$, and calculates its valuation on each subcarrier $v_{k, m}^{a}(t)$.

\section{- Bidding:}

Because there are $M$ subcarriers, every user $k$ submits a set of bid $b_{k, m}(t)$ according to its valuation $v_{k, m}^{a}(t)$ on subcarrier $m$. Furthermore, it is also related to the reported data rate $r_{k, m}(t)$ other than its true rate $r_{k, m}^{a}(t)$. Thus, it can also be expressed as:

$b_{k, m}(t)=f_{b}\left(v_{k, m}^{a}(t)\right)=v_{k, m}(t)=\log \left(1+\frac{r_{k}^{\prime}(t)+r_{k, m}(t)}{\left(t_{c}-1\right) \overline{R_{k}(t)}}\right)$

- Allocation and payment:

After collecting all bids from these $K$ users, for every subcarrier $m$, the auctioneer determines the allocation results $x_{k, m}^{*}(t)$ according to the greedy MC PF scheme, and calculates the payment $p_{k, m}(t)$ to be paid by user $k$ according to VCG auction, i.e.,

$$
\begin{aligned}
& x_{k, m}^{*}(t)= \\
& \underset{\left\{x_{k, m}(t)\right\}}{\arg \max }\left(\sum_{k=1}^{K} b_{k, m}(t)+\sum_{j \neq k} \log \left(1+\frac{r_{j}(t)}{\left(t_{c}-1\right) \cdot \overline{R_{j}(t)}}\right)\right) \cdot x_{k, m} \\
& p_{k, m}(t)=\max _{\left\{x_{k, m}(t)\right\}}\left\{\sum_{j \neq k} b_{k, m}(t) \cdot x_{k, m}(t)\right\}-\sum_{j \neq k} b_{j, m}(t) \cdot x_{j, m}^{*}(t)
\end{aligned}
$$

The first term of (9) is the maximum aggregated valuation that other users can derive if user $k$ does not participate in the auction, and the second term is the sum of aggregated valuation of the other users except user $k$ under optimal resource allocation results $x_{k, m}^{*}(t)$ in the presence of user $k$. Thus the payment paid by each user corresponds to the loss of declared valuation it imposes to the others through its presence.

It should be noticed that one subcarrier can only be occupied by just one user. Thus, the sealed-bid second-price auction [4], which is a special case of VCG auction, can be used to simplify the process of calculating allocation and payment results. Let $k_{m}^{*}(t)$ be the user chosen by the auctioneer to occupy subcarrier $m,(8)$ and (9) can be replaced by the following expressions:

$$
\begin{gathered}
k_{m}^{*}(t)=\underset{k}{\arg \max }\left(b_{k, m}(t)+\sum_{j \neq k} \log \left(1+\frac{r_{j}(t)}{\left(t_{c}-1\right) \cdot \overline{R_{j}(t)}}\right)\right) \\
p_{k, m}(t)=\left\{\begin{array}{cc}
\max _{j \neq k_{m}^{*}(t)} b_{j, m}(t) & k=k_{m}^{*}(t) \\
0 & k \neq k_{m}^{*}(t)
\end{array}\right.
\end{gathered}
$$

Notice that the auction-based algorithm is suitable when the users come and go in a dynamic situation, because the VCG mechanism can prevent users from cheating, and the auctioneer allocates the resource to the bidder with the highest bid. Thus, when a new user enters the network and submits bid, the auctioneer can still process this information according to the proposed algorithm. In terms of computational complexity of the algorithm, we can see that after collecting all bids from the $K$ users, for every subcarrier $m$, the auctioneer determines the allocation results according to Eq. (10), and calculates the payment to be paid by user $k$ according to Eq. (11). Thus, for a system with $K$ users and $M$ subcarriers, the computational complexity is $K M$.

\section{Simulations}

In this section, we present our simulation results on the model used in [12]. The entire system bandwidth is $20 \mathrm{MHz}$, which is divided into 256 subcarriers. The wireless channel is also modelled as a six-path frequency-selective Rayleigh fading channel. Each path is simulated by Clark's fading model and suffers from different Rayleigh fading with the maximum Doppler frequency of $30 \mathrm{~Hz}$, which corresponds to the average speed of $4.5 \mathrm{~km} / \mathrm{h}$ for the carrier frequency of $5 \mathrm{GHz}$. The maximum BER requirement is $10^{-5}$.

We verify the intuition that the users will be penalized if they lie about their resource requirements by exaggerating their own valuations. In this simulation, we compare the throughput as well as the payments paid by users under two scenarios: (1) no user is lying about its valuation, and (2) only User 5 is lying about its valuation, but the others are 
telling the truth. Table I shows the efficiency and fairness performance, as well as the payments, for the two cases.

From this table, we can see that when all users reveal the true valuations of the subcarriers, the resources are allocated among users efficiently and in a fair manner. However, when User 5 exaggerates its valuation, its throughput is improved by $94.39 \%$, but the payment to be paid is also significantly increased by $1525.88 \%$. Furthermore, the throughput of any other user is decreased dramatically. Thus, the fairness performance also deteriorates due to the selfish behaviour of User 5. Now, it becomes clear that, by using the VCG-based auction scheduling, the lying of selfish users is penalized through a significantly increased payment. Thus, a rational user will not lie about its valuation of subcarriers because the increased cost cannot be compensated by the improved throughput. Consequently, with the proposed auction-based scheduling scheme, selfish users will be enticed to report their channel conditions and corresponding valuation of channels honestly. Moreover, its bid is just its true valuation of the channels, i.e., $b_{k, m}(t)=v_{k, m}^{a}(t)$. Thus, PF can be achieved in non-cooperative multiuser OFDM system as that in a cooperative environment.

\section{CONCLUSIONS}

In this paper, we extend our previous research about non-cooperative scheduling in TDMA system in two aspects. Firstly, we focus on the problem of how to allocate resource with $\mathrm{PF}$, while in [1] the fairness performance is not considered. Secondly, we study this non-cooperative resource allocation in a multiuser OFDM environment instead of a single-carrier TDMA system considered in [1]. We propose an auction-based scheduling algorithm, which combines the merits of the VCG auction and the greedy MC $\mathrm{PF}$ algorithm, to ensure that wireless users truthfully declare their resource requirements and achieve a low-complexity $\mathrm{PF}$ scheduling in a non-cooperative multi-user OFDM system. Through simulations, we find that users lying about their resource requirements are severely penalized by very high payments so that they should rather declare true valuation of subcarriers to the scheduler. We also observe that a conventional PF scheduling scheme heavily depends on the truthfully reporting from wireless users, then it will result in significantly worse performance when users dishonestly report their requirements, because it does not provide a mechanism to penalize selfish users for misusing resources. In summary, the proposed auction-based scheduling can be used efficiently in non-cooperative situation to realize proportional fairness.

\section{REFERENCES}

[1] Z. Kong, Y. K. Kwok, and J. Wang, "On the Impact of Selfish Behaviors in Wireless Packet Scheduling," In Proceedings of IEEE ICC 2008, pp.3253-3257, Beijing, China, May 2008.

[2] A. Jalali, R. Padovani, and R. Pankai, "Data Throughput of CDMA-HDR a High Efficiency-High Data Rate Personal Communication Wireless System," In Proceeding of IEEE VTC2000-Spring, vol. 3, pp. 1854-1858, Tokyo, Japan, Jan. 2001.

[3] J. Jang and K. B. Lee, "Transmit Power Adaptation for Multiuser OFDM Systems," IEEE J. Select. Areas Commun., vol. 21, no. 2, pp.171-178, Feb. 2003.

[4] V. Krishna, Auction Theory, Academic Press, 2002.

[5] J. Sun, E. Modiano, and L. Zheng, "Wireless Channel Allocation Using an Auction Algorithm," IEEE $J$. Select. Areas Commun., vol. 24, no. 5, pp.1085-1096, May 2006.

[6] J. Huang, Z. Han, M. Chiang, and H.V. Poor, "Auction-based Distributed Resource Allocation for Cooperative Transmission in Wireless Networks," In Proceedings of IEEE Globecom 2007, pp.4807-4812, Washington, DC, USA, Nov. 2007.

[7] F. Fu and M. van der Schaar, "Noncollaborative Resource Management for Wireless Multimedia Applications Using Mechanism Design," IEEE Trans. Multimedia, vol. 9, no. 4, pp. 851-868, June 2007.

[8] S. Gandhi, C. Buragohain, L. Cao, H. Zheng, and S. Suri, "A General Framework for Wireless Spectrum Auctions," In Proceedings of DySPAN 2007, pp.22-33, Dublin, Ireland, Apr. 2007.

[9] S. Pal, S.R. Kundu, M. Chatterjee, and S.K. Das, "Combinatorial Reverse Auction-based Scheduling in Multirate Wireless Systems," IEEE Trans. Computers, vol. 56, no. 10, pp. 1329-1341, Oct. 2007.

[10] S. Han and Y. Han, "A Competitive Fair Subchannel Allocation for OFDMA System Using an Auction Algorithm," In Proceedings of IEEE VTC'07-Fall, pp.1787-1791, Baltimore, MD, USA, Sept.-Oct. 2007.

[11] S. de Vries and R. Vohra, "Combinatorial Auctions: A survey," INFORMS Journal on Computing, pp. 284-309, Mar. 2003.

[12] Z. Kong, J. Wang, and Y. K. Kwok, "A New Cross Layer Approach to QoS-Aware Proportional Fairness Packet Scheduling in the Downlink of OFDM Wireless Systems," In Proceedings of IEEE ICC 2007, pp. 5695-5700, Glasgow, Scotland, June 2007.

[13] H. Kim and Y. Han, "A Proportional Fair Scheduling for Multicarrier Transmission Systems," IEEE Commun. Letters, vol. 9, pp. 210-212, Mar. 2005.

Table I: Comparisons of throughput, fairness and payments in the two cases.

\begin{tabular}{|c|c|c|c|c|c|c|}
\hline \multirow[b]{2}{*}{ User } & \multicolumn{2}{|c|}{ (1) No users lying } & \multicolumn{2}{|c|}{ (2) Only User 5 lying } & \multirow{2}{*}{$\begin{array}{l}\Delta \text { Throughput } \\
(\%)\end{array}$} & \multirow{2}{*}{$\begin{array}{l}\Delta \text { Payment } \\
(\%)\end{array}$} \\
\hline & $\begin{array}{l}\text { Throughput } \\
\text { (Kbps) }\end{array}$ & Payment & $\begin{array}{l}\text { Throughput } \\
\text { (Kbps) }\end{array}$ & Payment & & \\
\hline 1 & 6.202 & 5.777 & 4.696 & 80.07 & $-24.28 \%$ & $1386.01 \%$ \\
\hline 2 & 6.536 & 6.050 & 4.835 & 82.02 & $-26.03 \%$ & $1355.70 \%$ \\
\hline 3 & 5.477 & 5.527 & 3.952 & 72.60 & $-27.84 \%$ & $1313.33 \%$ \\
\hline 4 & 5.879 & 4.870 & 4.781 & 65.80 & $-18.68 \%$ & $1351.75 \%$ \\
\hline 5 & 6.953 & 7.013 & 13.516 & 107.01 & $+94.39 \%$ & $1525.88 \%$ \\
\hline
\end{tabular}

